\title{
Phenotypic plasticity of Myzus persicae (Hemíptera: Aphididae) raised on Brassica oleracea L. var. acephala (kale) and Raphanus sativus L. (radish)
}

\author{
Fernanda Borja Peppe and Cecília Lomônaco \\ Instituto de Biologia, Universidade Federal de Uberlândia, Uberlândia, MG, Brazil.
}

\begin{abstract}
The study of variability generated by phenotypic plasticity is crucial for predicting evolutionary patterns in insect-plant systems. Given sufficient variation for plasticity, host race formation can be favored and maintained, even simpatrically. The plasticity of size and performance (assessed by the lifetime fitness index $r_{m}$ ) of six clones of Myzus persicae was tested, with replicates allowed to develop on two hosts, kale (Brassica oleracea var. acephala) and radish (Raphanus sativus). The clones showed significant variability in their plasticity. Reaction norms varied through generations and negative genetic correlation, although not significant, tend to increase with the duration of host use. The lack of plasticity in lifetime fitness among generalist clones occurred as an after-effect of the highly plastic determinants. Significant morphological plasticity in host used was observed, but no variation in the plastic responses (GxE interaction) was detected. Strong selection for a larger size occurred among individuals reared on radish, the most unfavorable host. Morphological plasticity in general body size (in a multivariate sense) was not linear related to fitness plasticity. These observations suggest that a high potential for the evolution of host divergence favors host race formation.
\end{abstract}

Key words: quantitative genetics, genetic correlation, morphological plasticity, phenotypic correlation, physiological plasticity, trade-offs.

Received: January 19, 2001; accepted: December 10, 2002.

\section{Introduction}

Phenotypic variation in natural populations is influenced by genetic variability but is also environmentally dependent (Schlichting, 1986; Scheiner, 1993; Via et al., 1995). Such environmentally-modulated plasticity may have an important role in the evolution of insect/plant interactions (Schlichting, 1986; Mopper, 1996). The plasticity of an insect in relation to its host allows the production of a superior phenotype without major genetic changes (Via, 1990; Thompson, 1991). Several phytophagous insects are known for their ability to use hosts that are morphologically and chemically distinctive (Gold, 1979; Fry, 1989). The performance of plastic organisms can be explained ecologically by their capacity to make physiological, morphological and behavioral adjustments in response to the nutritional, toxicological and anatomical features of their host plants.

Morphological plasticity, including the absence of a response to the environment, does not necessarily mean the lack of an ability to explore multiple host plants. Morphological effects may be mediated through marked plasticity

Send correspondence to Cecília Lomônaco. Instituto de Biologia, Universidade Federal de Uberlândia, 38400-902 Uberlândia, MG, Brazil. E-mail: lomonaco@ufu.br. of physiological characters (Schlichting, 1986). In some cases, morphological plasticity occurs as a byproduct of developmental systems with a maladapted archetype (Schlichting and Pigliucci, 1995), and therefore does not represent a mechanism by which relative fitness is maintained in response to environmental variation (Thompson, 1991).

Given sufficient variation in the plastic responses for a particular trait (variation in reaction norms), plasticity can evolve independently of the mean trait (Scheiner, 1993). However, if the pressures on each host are increased or maintained, the costs for plasticity can become very high and lead to trade-offs (Via and Lande, 1985; Sterns, 1989). Trade-offs constitute the fundamental basis for host race formation (Joshi and Thompson, 1995; Mackenzie, 1996). Therefore, knowledge of the developmental patterns underlying phenotypic variation is crucial for understanding evolutionary mechanisms, particularly specialization.

This study describes the morphological and physiological plastic responses of Myzus persicae clones under distinct environmental regimes imposed by the host plant characteristics. The effects of each environment on the genetic structure of the population and the relevance of 
phenotypic plasticity to the evolution of host race formation are discussed.

\section{Material and Methods}

\section{Collection and maintenance of clones}

Adult female Myzus persicae were collected at several locations in the city of Uberlândia (MG, Brazil), from February to April, 1998, to establish laboratory parthenogenetic clones. These clones were collected from distinct hosts (broccoli, kale, radish, arugula and Chinese cabbage) kept in order to ensure genetic variability. Stock cultures were maintained on Chinese cabbage leaves and in plastic containers in a refrigerator $\left(15^{\circ} \mathrm{C}\right)$. Chinese cabbage was selected as the host for stock cultivation because of its easy conservation and extended durability under refrigeration.

\section{Physiological plasticity}

The experiments were done during March, 1998, in experimental gardens at the Federal University of Uberlândia. Brassica oleracea L. var. acephala (kale) and Raphanus sativus L. (radish) were cultivated as the hosts to be tested. Adult females from each clone of M. persicae, were placed individually in clip cages attached to leaves of the hosts in order to initiate parthenogenetic descendents. From each generation produced, some individuals were chosen at random to establish parthenogenetic lineages. These individuals were monitored for their developmental time (from birth to the first reproductive day) and daily fecundity (number of nymphs produced each day). Alata females were discarded. Three generations were analyzed, giving a total of 30 individuals per clone from each host.

A life time index of fitness was calculated using the formula $\mathrm{r}_{\mathrm{m}}=(0.745 / \mathrm{dt}) * \log _{\mathrm{e}}(\mathrm{ft})$, where $\mathrm{dt}$ is the developmental time and $\mathrm{ft}$ is the number of nymphs produced by the time that the parent aphid is exactly two dt days old (Wyatt and White, 1977). The values for $r_{m}$, daily fecundity and developmental time were examined using analysis of variance in order to obtain the species' plastic potential and its variability among clones (Via and Lande, 1985). The causal components of variation were estimated from the mean square values in a two-way mixed model ANOVA table performed across environments (hosts). The use of cloned individuals can facilitate the estimation of genetic and environmental components of plasticity, although it does not allow the partitioning of genetic variances into additive and non-additive effects (Via, 1990). Norms of reaction were constructed for each character analyzed and genetic correlations were assessed to verify whether there were trade-offs (Falconer, 1989).

\section{Morphological plasticity}

Aphids were preserved in 70\% ethanol prior to being mounted for morphometric analysis under a microscope equipped with a micrometer. Five morphological features were measured in 15 adult individuals from each clone from both environments (hosts). These characters were: ultimate rostral segment length (UR), distance between the siphunculi (SS) and lengths of the right antennal segment III (A), hind tibia (HT), and siphunculus (S).

Principal Component Analysis was used to assess the nature and magnitude of variation in the morphological characters, and an index of general body size (in a multivariate sense) was then estimated using a correlation matrix of the original characters (Manly, 1994). The plasticity and causal components of variation were analysed as described above for the physiological characters. The genetic correlation for general size across environments was estimated using the scores of the first principal component. The phenotypic and genetic correlations between physiological and morphological characters were also assessed in order to determine the extent to which two particular characters were influenced by the same genes (Falconer, 1989). These correlations were estimated using the Pearson correlation coefficient. All statistical analyses were done using the computer software package SYSTAT for Windows, version 9.0 (Systat, Evaston, Ill, USA).

\section{Results}

\section{Plasticity of fitness and its components}

The average values and standard errors for the traits studied on each host are given in Table 1. Analysis of variance showed a significant difference in the average performance (genetic variability) among genotypes, as assessed by the fitness lifetime measurements in both environments $(\mathrm{F}=5.581, \mathrm{p}<0.0001)$. Clones responded phenotypically to the environmental conditions and this source of variation explained most of the total variance $(\mathrm{F}=59.854$; $\mathrm{p}<0.0001)$. Significant variability in quantitative plasticity among genotypes (genotypic $\mathrm{x}$ environment interaction) was also present, indicating a different response for each genotype $(\mathrm{F}=7.474, \mathrm{p}<0.0001)$ (Table 2$)$.

The reaction norms that indicated the direction of variation (Figure 1) confirmed the variability and demonstrated the occurrence of trade-offs at a population level. Thus, the clones with the best performance on kale were, respectively, those with the worse fitness indices on radish. For other clones, the performances on both hosts were similar. The fitness values fluctuated through generations, and

Table 1 - Traits (average \pm standard errors) of Myzus persicae clones reared on kale (Brassica oleracea var. acephala) and radish (Raphanus sativus).

\begin{tabular}{lcc}
\hline & Radish & Kale \\
\hline Fitness (rm) & $0.12 \pm 0.01$ & $0.31 \pm 0.01$ \\
Fecundity & $5.71 \pm 0.18$ & $4.85 \pm 0.10$ \\
Size & $0.26 \pm 0.11$ & $-0.34 \pm 0.09$ \\
Developmental time & $6.60 \pm 0.11$ & $7.60 \pm 0.13$ \\
\hline
\end{tabular}


Table 2 - Two-way ANOVA for the fitness $\left(\mathrm{r}_{\mathrm{m}}\right)$ of Myzus persicae clones reared on kale (Brassica oleracea var. acephala) and radish (Raphanus sativus).

\begin{tabular}{lcccrc}
\hline Source & SS & DF & MS & F & p \\
\hline Clone & 0.787 & 5 & 0.157 & 5.581 & $<0.0001$ \\
Host & 1.687 & 1 & 1.687 & 59.854 & $<0.0001$ \\
Clone x Host & 1.054 & 5 & 0.211 & 7.474 & $<0.0001$ \\
\hline
\end{tabular}
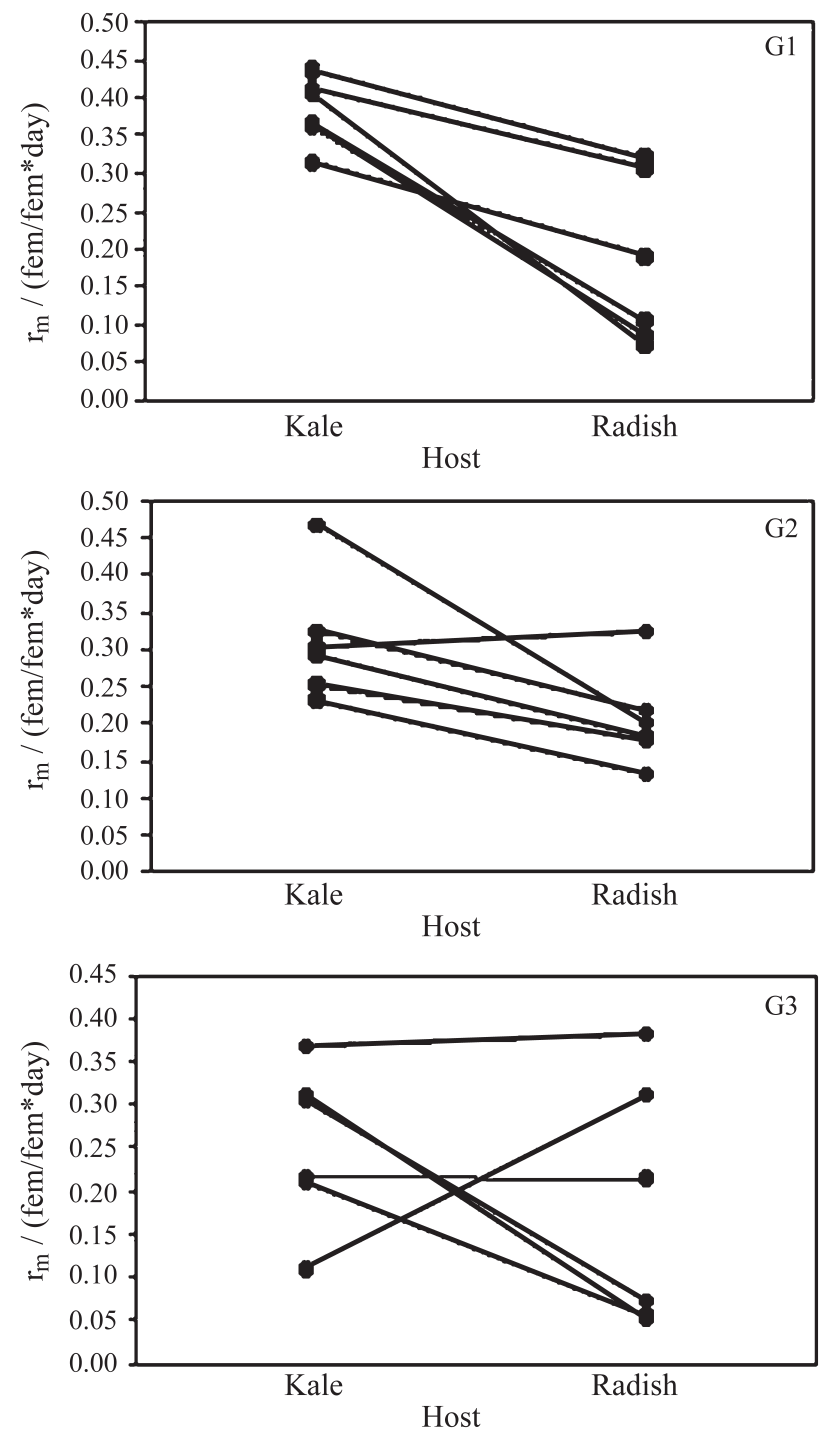

Figure 1 - Reaction norm for the fitness $\left(\mathrm{r}_{\mathrm{m}}\right)$ of Myzus persicae clones reared on kale (Brassica oleracea var. acephala) and radish (Raphanus sativus).

no consistent pattern of variation was detected among clones on the two hosts. Such instability modified the reaction norm diagrams over time, mainly because of the increases in total variability (Figure 2).

The relative contributions of the components of variation to each generation indicated by ANOVA showed a decrease in the variability caused by environmental

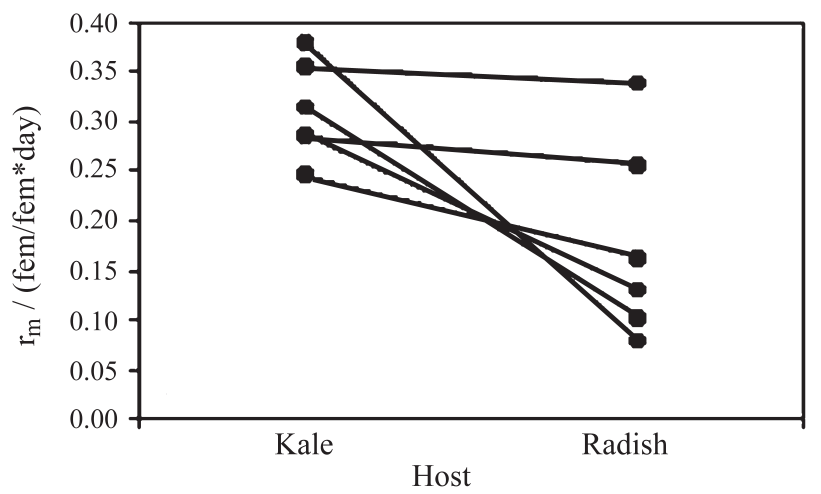

Figure 2 - Reaction norm for the fitness $\left(\mathrm{r}_{\mathrm{m}}\right)$ among three generations (G1, $\mathrm{G} 2, \mathrm{G} 3$ ) of Myzus persicae clones reared on kale (Brassica oleracea var. acephala) and radish (Raphanus sativus).

conditions, followed by an increase in variability due to genetic and genetic $\mathrm{x}$ environment influence (Table 3). Fecundity was significantly higher among individuals reared on radish than those reared on kale $(\mathrm{F}=12.013, \mathrm{p}=0.001)$. On the other hand, the developmental period was longer on kale than on radish $(F=35.393 ; p<0.0001)$. Some clones did not show plasticity for these components (Table 1).

\section{Plasticity of morphological traits}

All morphological traits were highly correlated so that a multivariate approach using PCA was satisfactory for reducing the dimensionality of the data (Manly, 1994). Since the signs and magnitudes of the first principal component were very similar, this component was interpreted as an index of general size, explaining $54.6 \%$ of the total variation (Table 4). Two-way ANOVA was done but the scores of the first principal components revealed no genetic variability among the clones and no significant interaction between clones and hosts (Table 5). However, environment (host) had a significant effect and explained most of the total variation for general size. All of the clones, except for one, tended to be larger when reared on radish than on kale.

Table 3 - Two-way ANOVA for fitness $\left(\mathrm{r}_{\mathrm{m}}\right)$ among three generations (G1, G2, G3) of Myzus persicae clones reared on kale (Brassica oleracea var. acephala) and radish (Raphanus sativus).

\begin{tabular}{lllllrc}
\hline $\begin{array}{l}\text { Genera- } \\
\text { tion }\end{array}$ & \multicolumn{1}{c}{ Source } & SS & DF & MS & F & p \\
\hline \multirow{2}{*}{ G1 } & Clone & 0.332 & 5 & 0.066 & 2.266 & 0.0540 \\
& Host & 0.918 & 1 & 0.918 & 31.326 & $<0.0001$ \\
& Clone x Host & 0.188 & 5 & 0.038 & 1.283 & 0.2780 \\
& Clone & 0.460 & 5 & 0.092 & 2.532 & 0.0300 \\
G2 & Host & 0.443 & 1 & 0.443 & 12.209 & 0.0010 \\
& Clone x Host & 0.286 & 5 & 0.057 & 1.575 & 0.1690 \\
& Clone & 0.510 & 5 & 0.102 & 5.712 & $<0.0001$ \\
G3 & Host & 0.123 & 1 & 0.123 & 6.874 & 0.0090 \\
& Clone x Host & 0.556 & 5 & 0.111 & 6.231 & $<0.0001$ \\
\hline
\end{tabular}


Table 4 - Two first principal components for morphometric measurements of adult female Myzus persicae reared on kale (Brassica oleracea var. acephala) and radish (Raphanus sativus).

\begin{tabular}{lcc}
\hline Morphometric & \multicolumn{2}{c}{ Components } \\
\cline { 2 - 3 } measurements & 1 & 2 \\
\hline $\mathrm{S}$ & 0.787 & 0.398 \\
$\mathrm{SS}$ & 0.656 & -0.534 \\
$\mathrm{~A}$ & 0.826 & 0.374 \\
$\mathrm{HT}$ & 0.859 & 0.115 \\
$\mathrm{UR}$ & 0.509 & -0.728 \\
$\begin{array}{l}\text { Variance explained } \\
\text { by components }\end{array}$ & 2.730 & 1.728 \\
Percent of total variance & & \\
explained (\%) & 54.6 & 22.6 \\
\hline
\end{tabular}

(A: right antennal segment III; HT: hind tibia; S: right siphunculi; SS: distance between the siphunculi; UR: ultimate rostral segment length).

Table 5 - Two-way ANOVA for the size (scores of the first component) of Myzus persicae clones reared on kale (Brassica oleracea var. acephala) and radish (Raphanus sativus)

\begin{tabular}{lrrrrl}
\hline Source & SS & DF & \multicolumn{1}{c}{ MS } & \multicolumn{1}{c}{ F } & p \\
\hline Clone & 8.984 & 5 & 1.797 & 2.082 & 0.0700 \\
Host & 20.519 & 1 & 20.519 & 23.781 & $<0.0001$ \\
Clone x Host & 4.633 & 5 & 0.927 & 1.074 & 0.3770 \\
\hline
\end{tabular}

\section{Genetic and phenotypic correlations}

The genetic correlation between general size and environments was not significant $(\mathrm{r}=0.126, \mathrm{p}=0.813)$, nor was size correlated with fecundity $\left(\mathrm{r}_{\text {radish lineages }}=0.975\right.$, $p=0.273 r_{\text {kale lineages }}=0.596, p=0.212$ ). Size was also not correlated with fitness in a specific environment $\left(\mathrm{r}_{\text {radish lineages }}=0.659, \mathrm{p}=0.155\right.$ and $\mathrm{r}_{\text {kale lineages }}=-0.344$, $p=0.505)$. Phenotypic correlations between size and fecundity and between size and fitness were significant for individuals raised on kale but not for those raised on radish.

\section{Discussion}

\section{Plasticity of host utilization}

Individual genotypes from the populations studied varied in their physiological responses according to the environmental conditions. The insects showed significant phenotypic plasticity for fitness when $B$. oleracea var acephala and $R$. sativus were used as hosts. There was also considerable variation in plasticity associated with the amount and direction of responses among genotypes. For more host-specialized genotypes, the cost of plasticity, i.e. the cost of maintaining the genetic and cellular machinery necessary to be plastic (Scheiner, 1993) is very high, and results in a gradual tendency toward specialization
(Rausher, 1988). Thus, an increase in the efficiency with which one host is used leads to a decrease in the efficient use of an alternative host. The gradual loss in the ability to use several hosts associated with the increased adaptation to a particular host indirectly expresses the selection for host races or biotype formation (Rausher, 1988).

The specialization of phytophagous insects generally depends on the ability to colonize a new host and on changes in the life history parameters of the population in a new environment. These factors may make gene flow between the original population and the newly founded population difficult (Futuyma and Moreno, 1988). Barriers to gene flow could not be investigated in M. persicae since this species reproduces only asexually at the latitude where the study was conducted. On the other hand, the conditioning of host choice, a process in which aphids tend to choose the host on which they were raised (Guldemond, 1990), could magnify the divergences between biotypes originating from disruptive selection (Pereira and Lomônaco, 2001).

Trade-offs in performance have been examined in the current paradigm to explain host specialization (Rausher, 1984). The lack of a significant negative genetic correlation for performance for the population studied does not mean that trade-offs were absent. Even small negative genetic correlations among traits simultaneously selected in distinct environments can promote evolutionary change (Rausher, 1988). Environmental modification can also produce positive genetic correlations that may counterbalance negative correlations (Service and Rose, 1985), and antagonistic pleiotropy may also be present without any detectable effect (Fry, 1993). The absence of negative genetic correlations may also mean that evolution of reaction norms is still in progress (Via and Lande, 1985).

The genetic correlation between the same traits in the two environments measures the degree of genetic independence (additivity) between these characters (Falconer, 1989) and allows the evaluation norms of reaction modification over time (Via, 1993). Nevertheless, this procedure does not assess the absence or presence of shared genetic control (independence of loci) since it includes the combined effects of allelic sensitivity (Schlichting and Pigliuci, 1993, 1995). Both of these models of gene-environmental interaction (e.g. pleiotropic and epistatic interactions) can contribute to the expression of plastic responses (Scheiner, 1993). Regardless of the mechanism responsible for generating fitness plasticity in M. persicae, the population is assumed to have sufficient variability in the character itself and in its ability to show plastic responses.

The presence of more generalist genotypes which possess much more flexible functional properties indicates that the costs of plasticity are not always high enough to curtail host use. To some extent, generalist genotypes were able to maintain a coherent metabolic integration on both hosts, without substantially compromising their fitness out- 
put or survival. Since the overall fitness of a genotype results from the integration of several characters, a less variable fitness index across environments can be a consequence of the genotype's highly plastic determinants. Indeed, the fitness components were very plastic across environments. The developmental period and fecundity varied between environments and across generations. For most of the M. persicae clones analyzed, a greater fecundity on radish was not accompanied by greater fitness on this host when compared with the fitness values on kale. This appears to be a consequence of the high mortality rates of the aphids when raised on radish. High mortality is therefore compensated for by high fecundity among generalists that may be adopting a strategy to maximize descendant survivorship (Dixon and Wellings, 1982). When plasticity contributes positively to fitness, it can be considered adaptive, and constitutes an important advantage in exploiting heterogeneous environments (Leclaire and Brandl, 1994).

\section{Plasticity of size}

The clones responded phenotypically to host quality. Although significant differences in size were observed, variation in the plastic responses (genetic $\mathrm{x}$ environment interaction) was not detected since all clones tended to be larger when reared on radish.

The variation in size among individuals reared on radish was smaller than among those raised on kale. This reduced variability indicated strong selection for size. Yet, why should survival on radish be conditioned to a specific size? If the costs of survivorship on radish are high, only larger individuals would be able to survive and reproduce. According to Dixon (1985), under stable conditions, fecundity and the size of aphid nymphs are positively correlated with their mother's size, although adverse conditions may sometimes alter this correlation (Grüber and Dixon, 1988).

An alternative hypothesis to explain the larger size of individuals reared on radish is that larger individuals also have larger feeding segments. In the particular case of aphids, several aspects of their hosts, such as the structure and abundance of trichomes on the leaf surface, (radish leaves have more trichomes than kale) are related with their modes of locomotion and the dimensions of the feeding apparatus (Moran, 1986). Constraints, such as the impact that the depth of phloem places on birth size have also been postulated as determinants in the selection for body size in aphids (Dixon et al., 1995). Therefore, if a larger ultimate rostral segment (URS) is necessary to increase fitness on radish, the smallest aphids would not be expected to survive. Characteristics that help an organism maintain its fitness are likely targets for selection (Via and Shaw, 1996). However, selection may not be operating on one character (URS) alone but on the whole phenotypic morphological expression of the organism (e.g. body size in the allometric sense).
As pointed out by Trussel (1996), caution must be exercised in evoking morphological plasticity as being responsible for microevolutionary change. Nevertheless, morphological divergence may have an important role in the mechanisms of sympatric speciation (Futuyma and Moreno, 1988).

\section{Genetic and phenotipic correlations}

Genetic correlations estimate the degree of independence of the expression of traits in two environments (Via and Lande, 1985). Thus, if characters are being influenced by an environmental stimulus, the phenotypic/genetic correlations among these characters would also be expected to change across environments (Schlichting, 1986). Host quality thus clearly influences the expression of fitness and size in M. persicae. Changes in the magnitude and direction of the genetic correlations appear to indicate different genetic mechanisms of character determination. This new genetic architecture may be caused by the expression of a new set of genes or the differential expression of the same genes, with pleiotropic effects on the characters measured (Holloway et al., 1990). Changes in the genetic correlations between traits in distinct environments have been reported in several studies (Rausher, 1984; Via, 1884a,b; Leclaire and Brandl, 1994).

The positive, significant phenotypic correlations between the characteristics analyzed among aphids raised on kale contrasted with the insignificant correlations among aphids raised on radish. This finding indicates that size and fitness did not covary uniformly across environments and reinforces the possibility that the genetic mechanisms controlling these traits are distinct, or at least not linearly related.

The environmental contribution to plasticity is thus not systematic for all characteristics, and genotypic effects may contribute to this diversification. Since the influence of allele sensitivity and loci independence cannot be separated, differences in at least one of these mechanisms among the genotypes would be sufficient to produce nonlinear correlated responses. These results indicate that host divergence provides a wide scope for evolution and favors the formation of host-specific races of M. persicae for kale and radish.

\section{Acknowledgments}

This project was partially supported by $\mathrm{CNPq}$, which provided a scholarship to F.B. Peppe. The authors thank Dr David Francis for reviewing the English of the manuscript.

\section{References}

Dixon AFG (1985) Aphid Ecology. Blackie, Glasgow, 157 pp.

Dixon AFG, Kindlmann P and Jarosik V (1995) Body size distribution in aphids: relative surface area of specific plant structure. Ecol Entomol 2:111-117. 
Dixon AFG and Wellings PW (1982) Seasonality and reproduction in aphids. Int J Inverteb Reprod 5:23-89.

Falconer DS (1989) Introduction to Quantitative Genetics. Longman, New York, 438 pp.

Futuyma DJ and Moreno G (1988) The evolution of ecological specialization. Annu Rev Ecol Syst 13:207-283.

Fry JD (1989) Evolutionary adaptation to host plants in a laboratory population of the phytophagous mite Tetranychus urticae Kock. Oecologia 81:559-565.

Fry JD (1993) The "general vigor" problem: can antagonistic pleiotropy be detected when genetic covariances are positive? Evolution 47:327-333.

Gold F (1979) Rapid host range evolution in a population of the phytophagous mite Tetranynchus urticae Kock. Evolution 33:791-802.

Grüber K and Dixon AFG (1988) The effect of nutrient stress on development and reproduction in an aphid. Entomol Exp Appli 47:23-30.

Guldlmond JA (1990) Choice of host plant as a factor in reproductive isolation of the aphid genus Cryptomyzus (Homoptera, Aphididae). Ecol Entomol 15:43-51.

Holloway GJ, Povey S and Sibly R (1990) The effect of new environment on adapted genetic architecture. Heredity 64:323330.

Joshi A and Thompson JN (1995) Trade-offs and the evolution of host specialization. Evol Ecol 9:82-92.

Leclaire M and Brandl R (1994) Phenotypic plasticity and nutrition in a phytophagous insect: consequences of colonizing a new host. Oecologia 100:379-385.

Mackenzie A (1996) A trade-off for host plant utilization in the black bean Aphis fabae. Evolution 50:155-162.

Manly BFJ (1994) Multivariate Statistical Methods. Chapman \& Hall, London, 215 pp.

Mopper S (1996) Adaptive genetic structure in phytophagous insect populations. Trends Ecol Evol 11:235-238.

Moran NA (1986) Morphological adaptation to host plant in Uroleucon (Homoptera: Aphididae). Evolution 40:10441050.

Pereira C and Lomônaco C (2001) Plasticidade fisiológica e comportamental de Brevicoryne brassicae (Hemíptera: Aphididae) em duas variedades de Brassica oleraceae L. Neotrop Entomol 30:29-35.

Rausher MD (1984) Trade-offs in performance on different hosts: evidence from within and between-site variation in the beetle Deloyala guttata. Evolution 38:582-595.
Rausher MD (1988) Is coevolution dead? Ecology 69:898-901.

Scheiner SM (1993) Genetics and evolution of phenotypic plasticity. Annu Rev Ecol 24:35-68.

Schlichting CD (1986) The evolution of phenotypic plasticity in plants. Annu Rev Ecol Syst 17:667-693.

Schlichting CD and Pigliucci M (1993) Control of phenotypic plasticity via regulatory genes. Am Nat 142:366-379.

Schlichting CD and Pigliucci M (1995) Gene regulation, quantitative genetics and the evolution of reaction norms. Evol Ecol 9:154-168.

Service PM and Rose MR (1985) Genetic covariation among life-history components: the effect of novel environments. Evolution 39:943-945.

Sterns SC (1989) The evolutionary significance of phenotypic plasticity. Bioscience 39:436-445.

Thompson JD (1991) Phenotypic plasticity as a component of evolutionary chance. Trends Ecol Evol 6:246-249.

Trussel GC (1996) Phenotypic plasticity in an intertidal snail: the role of a common crab predator. Evolution 50:448-454.

Via S (1984a) The quantitative genetics of polyphagy in an insect herbivore. I. Genotype-environment interaction in larval performance on different host plant species. Evolution $38: 881-895$

Via S (1984b) The quantitative genetics of polyphagy in an insect herbivore. II. Genetic correlations in larval performance within and among host plants. Evolution 38:896-905.

Via S (1990) Ecological genetics and host adaptation in herbivorous insects: the experimental study of evolution in natural and agricultural systems. Annu Rev Entomol 35:421-446.

Via S (1993) Regulatory genes and reaction norms. Am Nat 142:374-377.

Via S, Gomulkiewicz R, De Jong G, Scheiner SM, Schlichting CD and Van Tienderen PH (1995) Adaptive phenotypic plasticity: consensus and controversy. Trends Ecol Evol 19:212217.

Via S and Lande R (1985) Genotype-environment interactions and the evolution of phenotypic plasticity. Evolution 39:505-522.

Via S and Shaw AJ (1996) Short-term evolution in the size and shape of pea aphids. Evolution 50:163-173.

Wyatt IJ and White PF (1977) Simple estimation of intrinsic increase rates for aphids and tetranynchid mites. J Appl Ecol 14:757-766. 